\title{
Enantioselective Tertiary Electrophile (Hetero)Benzylation: Pd-Catalyzed Substitution of Isoprene Monoxide with Arylacetates
}

\author{
Michael G. J. Doyle, Alexis L. Gabbey, Wesley McNutt, Rylan J. Lundgren \\ Department of Chemistry, University of Alberta, Edmonton, Alberta, T6G 2G2, Canada \\ rylan.lundgren@ualberta.ca
}

\begin{abstract}
The enantioselective generation of quaternary carbon centers remains challenging but is of growing importance for the preparation of functional molecules. Transition metal catalyzed allylic alkylations of tertiary electrophiles have provided access to these substructures but remain generally incompatible with organometallic benzyl nucleophiles. In this study we demonstrate that electrondeficient arylacetates can serve as benzyl nucleophile surrogates to generate enantioenriched acyclic molecules containing a quaternary carbon center via a two-step substitution-decarboxylation process using isoprene monoxide. The reaction gives products typically in $>90 \%$ ee using a commercially available catalyst system and tolerates an array of electron-withdrawing functional groups on the arylacetate moiety. The lactone intermediate generated by the initial substitution reaction can be used in further stereoselective transformations to prepare molecules with acyclic vicinal quaternary stereocenters.
\end{abstract}

Stereogenic quaternary carbon centers are found in many bioactive molecules and pharmaceuticals. ${ }^{[1]}$ It is becoming more common that clinical candidates feature these units as medicinal chemistry programs seek out targets with increased three-dimensional structure. ${ }^{[2]}$ The catalytic, enantioselective preparation of acyclic quaternary carbon centers is made difficult by the steric encumbrance of substrates, the potential for poor orbital overlap between reactants, and the necessity for catalysts to differentiate between three non-hydrogen groups in molecules with high conformational freedom. Despite these challenges, the field of enantioselective synthesis of acyclic molecules bearing quaternary carbon centers has flourished thanks to an increased understanding of reaction mechanisms, new families of chiral catalysts, and improved tactics to generate reactive intermediates under mild conditions. ${ }^{[3]}$

Current state-of-the-art methods for the preparation of stereogenic quaternary carbon units include transition metal catalyzed allylic substitutions. ${ }^{[4]}$ Despite rapid advances in the areas of metalcatalyzed allylic alkylations using both hard ${ }^{[3,5]}$ and soft ${ }^{[6],[7]}$ carbon nucleophiles, certain structural classes of quaternary carbon units remain inaccessible by these approaches. Currently, the direct enantioselective benzylation of tertiary allylic electrophiles with main-group organometallics remains 
unknown. ${ }^{[8]}$ In this regard, it would be desirable to develop new approaches to enantioselective allylic benzylations where surrogates for hard $\mathrm{C}\left(\mathrm{sp}^{3}\right)$-nucleophiles are used. Alternative strategies for the enantioselective (hetero)benzylation of secondary allylic electrophiles include the use of deprotonated Lewis acid stabilized methyl azaheterocycles in combination with a strong base, ${ }^{[9]}$ benzylic deprotonation of $\operatorname{Cr}(\mathrm{CO})_{3}$ activated toluenes followed by decomplexation, ${ }^{[10]}$ fragmentation of 4-benzyldihydropyridines with photoredox co-catalysts, ${ }^{[11]}$ and the use of electron-rich diene nucleophiles that can undergo aromatization upon allylation (Fig 1a). ${ }^{[12]}$ A recent report established 4-benzyldihydropyridines can be used to generate quaternary benzylated stereocenters using $\alpha$-aryl allylic carbonate electrophiles; however enantioselectivities remain modest (generally $\sim 80 \%$ ee). ${ }^{[13]} \mathrm{We}$ previously developed a decarboxylative approach towards the enantioselective benzylation of secondary allylic electrophiles using arylacetic acids as pronucleophiles. ${ }^{[14]}$ In these reactions, decarboxylation occurs after stereoselective allylation. We questioned whether this concept could be expanded for the benzylation of tertiary allylic electrophiles, focusing on isoprene monoxide as it is an archetypal electrophile and is widely used in complex molecule synthesis. ${ }^{[15]}$ Here we report that Trosttype chiral Pd catalysts facilitate the enantioselective allylic alkylation of (hetero)arylacetate esters to generate lactone intermediates (Fig 1b). Subsequent hydrolysis and decarboxylation of the lactone generates acyclic products with chiral benzylated quaternary stereocenters, typically in $>90 \%$ ee. The ester group of the arylacetate acts as a traceless modifying group to tune reactivity. In addition, the lactone intermediates can be converted into acyclic products with vicinal quaternary stereocenters in a stereodivergent manner by an allylation-reductive ring-opening sequence (Fig 1b).
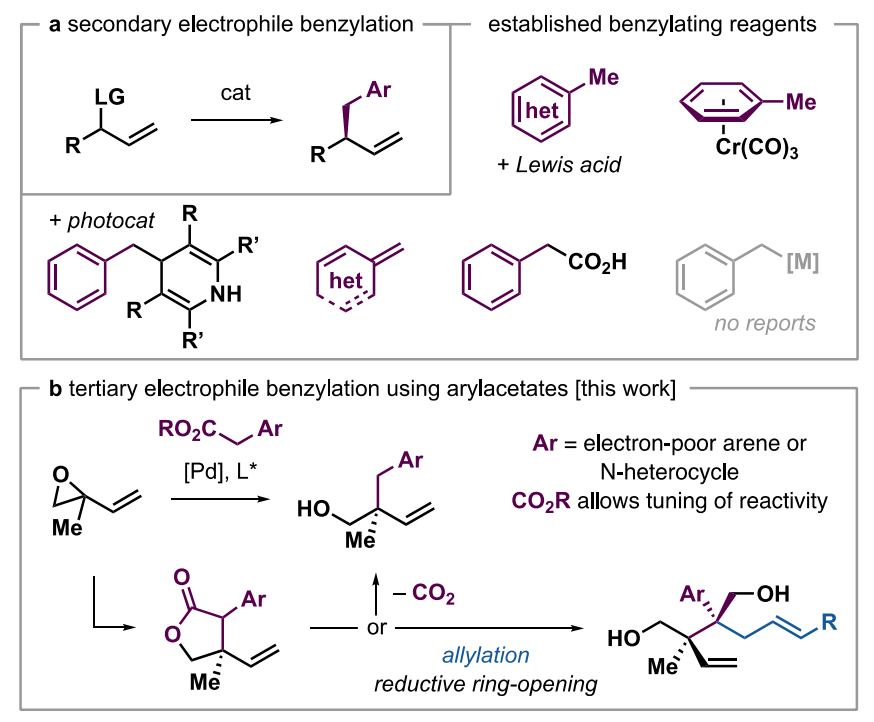

Figure 1. [a] Overview of approaches for allylic alkylation using benzylic nucleophiles with secondary electrophiles, [b] this study showing an arylacetate surrogate approach for the benzylation of isoprene monoxide. 
Reaction conditions and benzylic nucleophile surrogates were broadly examined with the aim of promoting enantioselective tertiary electrophile benzylation. It was ultimately found that the combination of $2.5 \mathrm{~mol} \%\left[\mathrm{Pd}(\text { cinnamyl) } \mathrm{Cl}]_{2}\right.$ and $5.5 \mathrm{~mol} \%$ Nap-Trost ligand catalyzes the enantioselective addition of the 2,2,2-trifluoroethyl (TFE) ester of 4-cyanophenylacetate to isoprene monoxide to generate lactone 1 in $60 \%$ yield and $94 \%$ ee (Fig 2). Lactone 1 is formed as an inconsequential $\sim 2: 1$ mixture of diastereomers and undergoes thermal decarboxylation to give the benzylated quaternary stereocenter containing product 2 in $72 \%$ isolated yield without the erosion of product ee (94\%). Other ligand classes, including bisphosphines, phosphoramidites, and Phox ligands were inferior to Trost-type ligands (Fig 2a). The corresponding ethyl ester of 4-cyanophenylacetate, which reacted at a slower rate, led to formation of $1 \mathrm{a}$ in $36 \%$ yield and $99 \%$ ee. Reducing the equivalents of the TFE-ester from two to one or reducing the catalyst loading from $5 \mathrm{~mol} \%$ to $2 \mathrm{~mol} \%$ also slowed reaction rates and gave lower terminal yields but slightly increased enantioselectivities compared to the standard conditions (Fig 2b, 53\% yield, $96 \%$ ee and $40 \%$ yield, $98 \%$ ee respectively). The use of $\mathrm{Cs}_{2} \mathrm{CO}_{3}$ instead of $\mathrm{DBU}$ as the base led to faster reactions with smooth conversion to product, but at the expense of enantioselectivity $(73 \%$ yield, $67 \%$ ee). In general, conversion of isoprene monoxide was $>95 \%$; with the remaining mass balance comprising of non-productive consumption of the epoxide (1,4-linear addition, diene and aldehyde formation, lactone allylation). These optimization studies highlight that reaction enantioselectivity is inversely related to rate, likely because the intermediate $\mathrm{Pd}$-allyl species requires time to undergo $\eta^{3}$ $\eta^{1}-\eta^{3}$ isomerization to interconvert between allyl diastereomers. ${ }^{[16]}$ Unfortunately, decreased reaction rates also lead to an increased amount of side product formation due to non-productive consumption of the epoxide. The optimized conditions therefore provide a compromise between selectivity and yield; however, in cases where $\geq 98 \%$ product ee is desirable, greater enantioselectivity can be achieved under modified conditions (Fig 2b). 


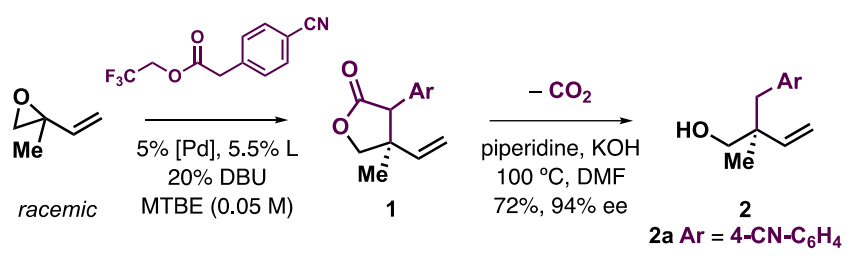

a impact of ligand [yield and ee of lactone 1]

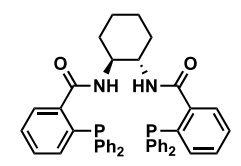

$52 \%$ yield, $91 \%$ ee

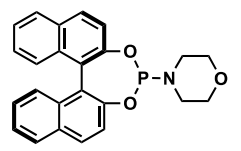

$<1 \%$ yield, n.d. ee

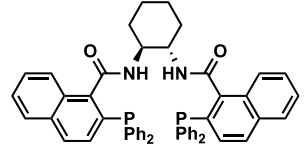

$60 \%$ yield, $94 \%$ ee

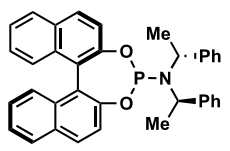

$<1 \%$ yield, n.d. ee ${ }^{\mathrm{a}}$

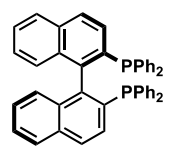

$17 \%$ yield, $40 \%$ ee

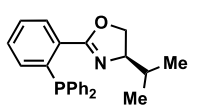

$6 \%$ yield, $20 \%$ ee

\begin{tabular}{|lcc|}
\hline & & \\
& b impact of reaction conditions [lactone 1] & \\
\cline { 2 - 3 } & yield (\%) & ee (\%) \\
no deviation & 60 & 94 \\
Et-ester instead of TFE-ester & 36 & 99 \\
Acid insted of TFE-ester & $<1$ & n.d. \\
1 equiv. ester instead of 2 & 53 & 96 \\
$2 \% \mathrm{Pd}, 2.2 \% \mathrm{~L}$ instead of 5 and $5.5 \%$ & 40 & 98 \\
$\mathrm{Cs}_{2} \mathrm{CO}_{3}$ instead of DBU & 73 & 67 \\
no base instead of DBU & $<1$ & n.d. \\
THF instead of MTBE & 50 & 83 \\
DCE instead of MTBE & 55 & 81 \\
dioxane instead of MTBE & 54 & 90 \\
& & \\
\end{tabular}

Figure 2. Optimization studies for the Pd-catalyzed enantioselective benzylation of racemic isoprene monoxide. Vinyl epoxide:arylacetate $=1: 2$. [a] Impact of ligand, [b] impact of reaction conditions. Yields determined by calibrated ${ }^{1} \mathrm{H}$ NMR, ee determined by chiral HPLC, lactone $\mathrm{dr}=66: 34$, reaction time 7$18 \mathrm{~h}$ at room temperature. $[\mathrm{Pd}]=\left[\mathrm{Pd}(\text { cinnamyl)Cl}]_{2},{ }^{\left[{ }^{2}\right.} 11 \mathrm{~mol} \%\right.$ ligand used.

At the outset of this study, it was not immediately certain whether lactone $\mathbf{1}$ would undergo decarboxylation in high yield. Generation of 2 requires ring-opening lactone hydrolysis and decarboxylation; ${ }^{[17]}$ both steps are reversible and decarboxylation is suppressed by water (Fig 3). ${ }^{[18]}$ It was found that the use of a polar aprotic solvent (DMF) at $100^{\circ} \mathrm{C}$ with an excess of $\mathrm{KOH}$ and piperidine provided optimal results (Fig 3, $78 \%$ yield of $\mathbf{2 a}$, complete retention of stereochemistry). The piperidine likely acts as a Lewis base to capture the liberated $\mathrm{CO}_{2}$, which stymies re-carboxylation of the anionic benzyl intermediate. ${ }^{[18]}$ 


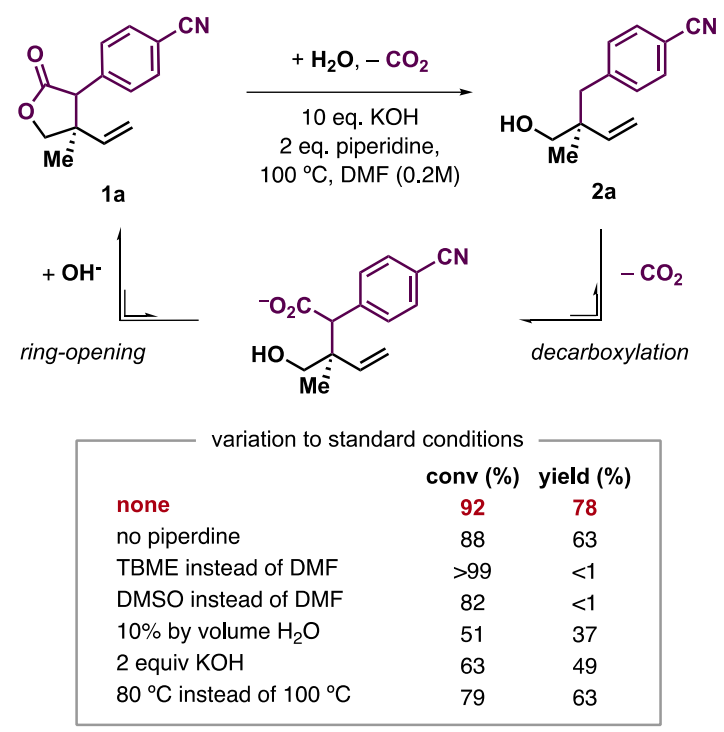

Figure 3. Effect of reaction parameters on lactone ring-opening decarboxylation. Yields determined by calibrated ${ }^{1} \mathrm{H}$ NMR, reaction time $40-48 \mathrm{~h}$.

The net enantioselective decarboxylative benzylation of isoprene monoxide accommodates an array of electron-poor (hetero)arylacetates to give products 2 in $85-99 \%$ ee (Fig 4a). Benzylated products containing aryl cyano, nitro, carbonyl, sulfonyl, sulfonamide, trifluoromethyl, chloro, and pentafluorosulfanyl groups are tolerated (2a-2j). For arylacetates that are more electron-deficient than 4-cyanoarylacetate, the use of the corresponding ethyl ester in THF with $\mathrm{Cs}_{2} \mathrm{CO}_{3}$ as the base was found to give higher enantioselectivities (2b, $\mathbf{2 d}$, and $\mathbf{2 0}$, see $\mathrm{SI}$ for a comparison of conditions). The use of the ethyl ester of these substrates decreases the acidity of the substrate in comparison to the TFE-ester, which in turn decreases the concentration of the reactive enolate nucleophile in solution and allows for a slower reaction that proceeds with higher enantioselectivity. Chiral products featuring electron-poor $\mathrm{N}$-heterocycles, including 2- and 4-pyridines, 2-pyrazine, and 2-quinoline (2k-2o) can be generated smoothly in good ee. While products with electron-neutral or electron-rich arenes are not directly amenable to the reaction (see the SI for unsuccessful substrates), these benzylated fragments can be easily obtained by, for example, reduction of the nitro group in $\mathbf{2 b}$ to generate aniline $\mathbf{2 p}$. Subsequent deamination gives benzyl product 2q.

The lactone intermediate from the first step does not require rigorous purification prior to decarboxylation. Filtration through a silica plug to remove base and the bulk of catalyst, followed by a solvent swap leads to similar overall two-step yields (see the SI for more details). The lactonization/decarboxylation approach provides access to benzylated products that are not accessible by known approaches. For example, direct benzylation of isoprene monoxide to prepare $2 \mathbf{r}$ with benzyl magnesium bromide or benzyl zinc reagents leads only to racemic products in modest yields. The use of Lewis acid complexation/deprotonation approaches for heterobenzylations that are viable for 
secondary electrophiles also fail when using tertiary epoxide electrophiles, for example to prepare $\mathbf{2 j}$ (Fig 4b). ${ }^{[30]}$ 

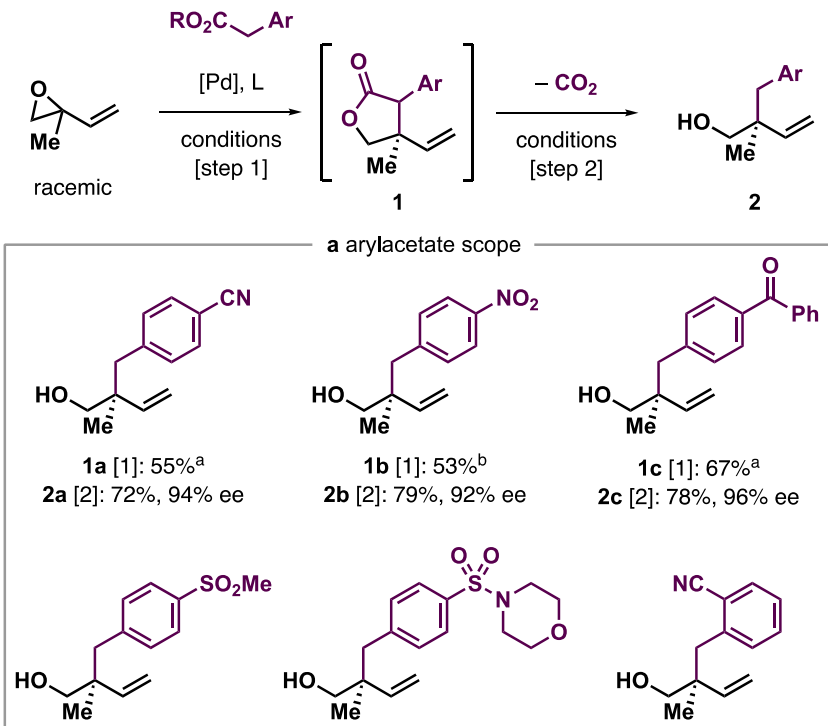

1d [1]: $51 \%$ b

2d [2]: $66 \%, 98 \%$ ee

a arylacetate scope

2

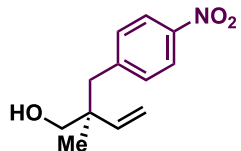

1b [1]: $53 \%$ b

2b [2]: $79 \%, 92 \%$ ee

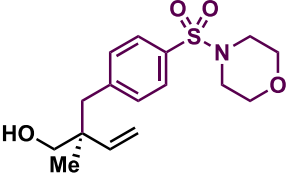

1e [1]: $40 \%^{\mathrm{a}}$

2e [2]: $70 \%, 94 \%$ ee<smiles>C=C[C@@](C)(CO)Cc1cc(C(F)(F)F)cc(C(F)(F)F)c1</smiles>

1h [1]: $49 \%^{c}$

2h [2]: $78 \%, 91 \%$ ee

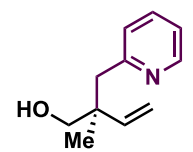

2k $40 \%, 96 \%$ ee $^{\mathrm{c}}$ [two step yield]

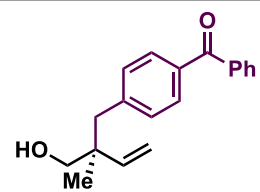

1c [1]: $67 \%^{\mathrm{a}}$ 2c [2]: $78 \%, 96 \%$ ee 1g [1]: $55 \%{ }^{\mathrm{c}}$

2g [2]: $71 \%, 94 \%$ ee<smiles>C=C[C@@](C)(CO)Cc1ccc(S(F)(F)F)cc1</smiles>

1j [1]: $55 \%$ c

2j [2]: $78 \%, 96 \%$ ee<smiles>C=C[C@](C)(CO)Cc1ccc2ccccc2n1</smiles>

2m 33\%, 85\% ee $\mathrm{ec}^{\mathrm{m}}$ [two step yield]

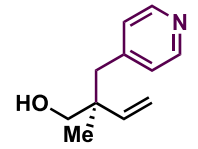

2n $36 \%, 92 \%$ eec [two step yield]

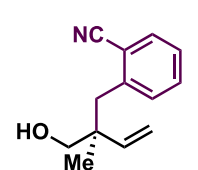

1f [1]: $45 \%^{\mathrm{a}}$ 2f [2]: $76 \%, 96 \%$ ee<smiles>C=C[C@](C)(CO)Cc1ccc(Cl)c(Cl)c1</smiles>

1i [1]: $52 \%$

2i [2]: $68 \%, 94 \%$ ee<smiles>C=C[C@@](C)(Cc1cnccn1)OC</smiles>

2l $40 \%, 87 \%$ ee $^{c}$ [two step yield]<smiles>C=C[C@@](C)(CO)Cc1ccnc(Cl)c1</smiles>

10 [1]: $45 \%$ b 2o [2]: $55 \%$, >99\% ee<smiles>C=C[C@@](C)(O)Cc1ccc(N)cc1</smiles>

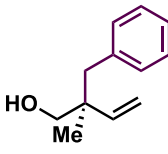

2p [3]: 95\%, 92\% ee

2q [4]: $81 \%, 92 \%$ ee

b comparison with organometallic nucleophiles

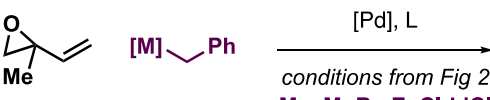

$$
\begin{aligned}
& \mathrm{M}=\mathrm{MgBr}, \mathrm{ZnCl} \cdot \mathrm{LiC}
\end{aligned}
$$

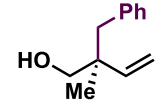

$2 q<40 \%$ yield, $<5 \%$ ee

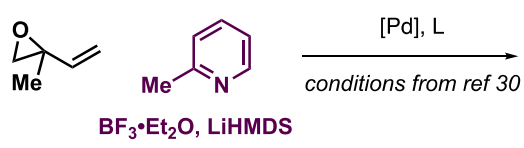


Figure 4. Scope of the Pd-catalyzed benzylation of isoprene monoxide. [a] Arylacetate nucleophile scope, [b] comparison with organometallic nucleophiles. $[\mathrm{Pd}]=[\mathrm{Pd}(\text { cinnamyl }) \mathrm{Cl}]_{2}, \mathrm{~L}=(R, R)$-Nap-Trost. Ring opening decarboxylation temperature $95-130^{\circ} \mathrm{C}$ in DMF. Step [3] nitro reduction, step [4] deamination, see SI for complete details. Yields are of isolated material unless otherwise noted. ${ }^{[a l} \mathrm{From}$ TFE-ester using $20 \mathrm{~mol} \%$ DBU in MTBE. ${ }^{[b]}$ From Et-ester using 60 mol\% $\mathrm{Cs}_{2} \mathrm{CO}_{3}$ in THF. ${ }^{[c]}$ From TFEester using $40 \%$ DABCO in MTBE.

In lieu of decarboxylation, the lactone generated by arylacetate substitution of isoprene monoxide can serve as an intermediate for other stereoselective transformations to generate different classes of acyclic molecules bearing quaternary carbons. Pd-catalyzed lactone allylation ${ }^{[19]}$ followed by $\mathrm{LiBH}_{4}$ mediated reductive ring-opening ${ }^{[20]}$ of $\mathbf{1 a}$ leads to diol products 4 that feature vicinal quaternary stereocenters in yields of $62-74 \%$ over two steps (Fig 5). ${ }^{[6 a, 21]}$ Access to either product diastereomer is possible by stereodivergent lactone allylation. ${ }^{[22]}$ When using $(R, R)$-Nap-Trost in the benzylation of isoprene monoxide, the use of the $(S, S)$ series of Trost-ligands in the subsequent lactone allylation leads to diol products in $\geq 97 \%$ ee and $\geq 94: 6 \mathrm{dr}(\mathbf{4 a}, \mathbf{4 b})$. The use of the $(R, R)$ series of Trost-ligands leads to the opposite diastereomer in $\geq 99 \%$ ee and $\geq 66: 34 \mathrm{dr}\left(\mathbf{4} \mathbf{a}^{\prime}, \mathbf{4} \mathbf{b}^{\prime}\right)$.

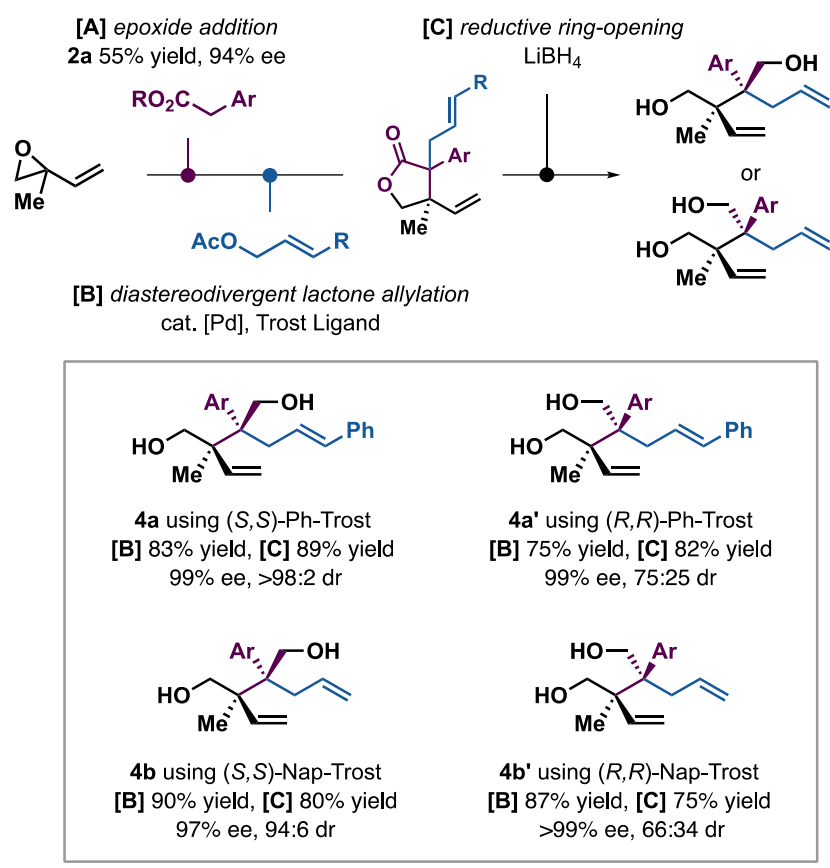

Figure 5. Diastereodivergent synthesis of targets with vicinal acyclic quaternary stereocenters by lactone allylation and reductive ring-opening.

Transition metal-catalyzed allylic alkylations are among the most robust methods to generate enantioenriched acyclic quaternary stereocenters. This work demonstrates that easy to prepare, bench stable arylacetates can act as surrogates for highly basic organometallic benzylic nucleophiles in allylic 
substitution reactions of tertiary electrophiles. After ring-opening decarboxylation of the lactone intermediates, (hetero)benzylated products can be obtained in generally $>90 \%$ ee. The lactone can also serve as an intermediate in the synthesis of additional classes of chiral acyclic allylated products via catalyst-controlled diastereodivergent reactions. This approach may find value in other classes of enantioselective reactions that are incompatible with classical organometallic benzylating reagents.

\section{Acknowledgements}

We thank NSERC Canada (RGPIN-2019-06050 and RGPAS-2019-00051 to R.J.L., CGS-M to M.G.J.D. USRA to A.L.G.) for support. Dr. Michael J. Ferguson (Alberta) is thanked for X-ray crystal structure determinations.

\section{Conflict of Interest}

The authors declare no conflict of interest.

\section{References}

[1] a. C. Li, S. S. Ragab, G. Liu, W. Tang, Nat. Prod. Rep. 2020, 37, 276-292; b. T. Ling, F. Rivas, Tetrahedron 2016, 72, 6729-6777.

[2] a. T. T. Talele, J. Med. Chem. 2020, 63, 13291-13315; b. F. Lovering, MedChemComm 2013, 4, 515-519; c. F. Lovering, J. Bikker, C. Humblet, J. Med. Chem. 2009, 52, 6752-6756.

[3] a. T. B. Wright, P. A. Evans, Chem. Rev. 2021 doi: 10.1021/acs.chemrev.0c00564; b. D. Pierrot, I. Marek, Angew. Chem. Int. Ed. 2020, 59, 36-49; c. J. Feng, M. Holmes, M. J. Krische, Chem. Rev. 2017, 117, 12564-12580; d. Y. Liu, S.-J. Han, W.-B. Liu, B. M. Stoltz, Acc. Chem. Res. 2015, 48, 740-751; e. J. P. Das, I. Marek, Chem. Commun. 2011, 47, 4593-4623; f. J. Christoffers, A. Baro, Quaternary Stereocenters: Challenges and Solutions for Organic Synthesis, Wiley-VCH, 2005.

[4] a. L. Süsse, B. M. Stoltz, Chem. Rev. 2021, 121, 4084-4099; b. B. M. Trost, J. E. Schultz, Synthesis 2019, 51, 1-30.

[5] a. A. H. Hoveyda, Y. Zhou, Y. Shi, M. K. Brown, H. Wu, S. Torker, Angew. Chem. Int. Ed. 2020, 59, 21304-21359; b. A. H. Cherney, N. T. Kadunce, S. E. Reisman, Chem. Rev. 2015, 115, 9587-9652; c. V. Hornillos, J.-B. Gualtierotti, B. L. Feringa, in Progress in Enantioselective Cu(I)-Catalyzed Formation of Stereogenic Centers (Ed.: S. R. Harutyunyan), Springer International Publishing, Cham, 2016, pp. 139; d. M. Fañanás-Mastral, R. Vitale, M. Pérez, B. L. Feringa, Chem. Eur. J. 2015, 21, 4209-4212; e. K. Hojoh, Y. Shido, H. Ohmiya, M. Sawamura, Angew. Chem. Int. Ed. 2014, 53, 4954-4958; f. M. Magrez, 
Y. Le Guen, O. Baslé, C. Crévisy, M. Mauduit, Chem. Eur. J. 2013, 19, 1199-1203; g. M. FañanásMastral, M. Pérez, P. H. Bos, A. Rudolph, S. R. Harutyunyan, B. L. Feringa, Angew. Chem. Int. Ed. 2012, 51, 1922-1925.

[6] a. J. C. Hethcox, S. E. Shockley, B. M. Stoltz, Angew. Chem. Int. Ed. 2018, 57, 8664-8667; b. S. E. Shockley, J. C. Hethcox, B. M. Stoltz, Angew. Chem. Int. Ed. 2017, 56, 11545-11548; c. B. M. Trost, C. Jiang, J. Am. Chem. Soc. 2001, 123, 12907-12908; d. P. Zhang, H. Le, R. E. Kyne, J. P. Morken, J. Am. Chem. Soc. 2011, 133, 9716-9719; For an alternative redox allylation approach see: e.J. Feng, V. J. Garza, M. J. Krische, J. Am. Chem. Soc. 2014, 136, 8911-8914.

[7] For the Ir-catalyzed enantioselective allylation of (non-benzylic) alkyl organozinc reagents using secondary electrophiles see: a. J. Y. Hamilton, D. Sarlah, E. M. Carreira, Angew. Chem. Int. Ed. 2015, 54, 7644-7647; b. D. A. Petrone, M. Isomura, I. Franzoni, S. L. Rössler, E. M. Carreira, J. Am. Chem. Soc. 2018, 140, 4697-4704.

[8] For achiral and diastereoselective benzylations with allylic electrophiles using organometallic reagents see: a. D. Pal, T. B. Wright, R. O'Connor, P. A. Evans, Angew. Chem. Int. Ed. 2021, 60, 29872992; b. V. Rosales, J. L. Zambrano, M. Demuth, J. Org. Chem. 2002, 67, 1167-1170.

[9] a. B. M. Trost, D. A. Thaisrivongs, J. Am. Chem. Soc. 2008, 130, 14092-14093; b. B. M. Trost, D. A. Thaisrivongs, J. Am. Chem. Soc. 2009, 131, 12056-12057; c. B. M. Trost, D. A. Thaisrivongs, J. Hartwig, J. Am. Chem. Soc. 2011, 133, 12439-12441; d. R. Murakami, K. Sano, T. Iwai, T. Taniguchi, K. Monde, M. Sawamura, Angew. Chem. Int. Ed. 2018, 57, 9465-9469.

[10] J. Mao, J. Zhang, H. Jiang, A. Bellomo, M. Zhang, Z. Gao, S. D. Dreher, P. J. Walsh, Angew. Chem. Int. Ed. 2016, 55, 2526-2530.

[11] H.-H. Zhang, J.-J. Zhao, S. Yu, J. Am. Chem. Soc. 2018, 140, 16914-16919.

[12] X.-J. Liu, C. Zheng, Y.-H. Yang, S. Jin, S.-L. You, Angew. Chem. Int. Ed. 2019, 58, 10493-10499.

[13] S. Xue, B. Limburg, D. Ghorai, J. Benet-Buchholz, A. W. Kleij, Org. Lett. 2021, 23, 4447-4451.

[14] P. J. Moon, Z. Wei, R. J. Lundgren, J. Am. Chem. Soc. 2018, 140, 17418-17422.

[15] a. B. M. Trost, N. G. Andersen, J. Am. Chem. Soc. 2002, 124, 14320-14321; b. M. Zeng, S. K. Murphy, S. B. Herzon, J. Am. Chem. Soc. 2017, 139, 16377-16388; c. L. Yang, T. Wurm, B. Sharma Poudel, M. J. Krische, Angew. Chem. Int. Ed. 2020, 59, 23169-23173; d. J. R. Patterson, L. R. Terrell, 
C. A. Donatelli, D. A. Holt, L. J. Jolivette, R. A. Rivero, T. J. Roethke, A. Shu, P. Stoy, G. Ye, M. Youngman, B. G. Lawhorn, J. Med. Chem. 2020, 63, 14867-14884.

[16] B. M. Trost, E. J. McEachern, J. Am. Chem. Soc. 1999, 121, 8649-8650.

[17] J. Q. Bond, D. Martin Alonso, R. M. West, J. A. Dumesic, Langmuir 2010, 26, 16291-16298.

[18] D. Kong, P. J. Moon, E. K. J. Lui, O. Bsharat, R. J. Lundgren, Science 2020, 369, 557-561.

[19] a. J. James, P. J. Guiry, ACS Catal. 2017, 7, 1397-1402; b. P. Meletis, M. Patil, W. Thiel, W. Frank, M. Braun, Chem. Eur. J. 2011, 17, 11243-11249; c. S. Aubert, T. Katsina, S. Arseniyadis, Org. Lett. 2019, 21, 2231-2235.

[20] K. Soai, A. Ookawa, J. Org. Chem. 1986, 51, 4000-4005.

[21] For recent examples and reviews on the catalytic synthesis of acyclic vicinal quaternary centers see ref $6 a$ and a. M. Isomura, D. A. Petrone, E. M. Carreira, J. Am. Chem. Soc. 2021, 143, 3323-3329; b. F. Zhou, L. Zhu, B.-W. Pan, Y. Shi, Y.-L. Liu, J. Zhou, Chem. Sci. 2020, 11, 9341-9365; c. L. Næsborg, L. A. Leth, G. J. Reyes-Rodríguez, T. A. Palazzo, V. Corti, K. A. Jørgensen, Chem. Eur. J. 2018, 24, 14844-14848; d. X. Liu, C. Zhao, R. Zhu, L. Liu, Angew. Chem. Int. Ed. 2021, doi: 10.1002/anie.202105594.

[22] For a review of diastereo-convergent asymmetric catalysis see: S. Krautwald, E. M. Carreira, J. Am. Chem. Soc. 2017, 139, 5627-5639. 